\title{
INTELIGENCIA EMOCIONAL: RESULTADOS PRELIMINARES SOBRE SU NATURALEZA Y CAPACIDAD PREDICTIVA A PARTIR DE UN ESTUDIO CORRELACIONAL EN MUESTRAS DE ESTUDIANTES DE SECUNDARIA
}

\author{
EMOTIONAL INTELLIGENCE: PRELIMINARY RESULTS ON ITS NATURE \\ AND PREDICTIVE VALIDITY BASED ON A CORRELATIONAL STUDY \\ IN A HIGH SCHOOL STUDENTS SAMPLE
}

\author{
José Miguel Mestre Navas*, Rocío Guil Bozal** y Rocio Mestre Moreno*** \\ Universidad de Cádiz*y**, Universidad de Sevilla***
}

\begin{abstract}
RESUMEN
Uno de los campos de aplicación de la inteligencia emocional (IE) está siendo desde hace pocos años el educativo. La posible vinculación entre la IE y la adaptación del alumnado al sistema educativo pasa por resolver algunas cuestiones a priori necesarias para los estudios de validez predictiva en contextos escolares. Por un lado, se necesita datos empíricos que muestren que estamos ante un constructo novedoso y, si esto fuese así, comprobar si la IE está relacionada con algunos criterios adaptativos sobre la adaptación escolar. Este estudio, es solo el principio de una serie de trabajos de los autores encaminados a mostrar que la IE es un concepto novedoso e independiente de otros de conceptos relacionados con la inteligencia y la personalidad; y por otro lado, se muestran algunos resultados relacionados con la adaptación.
\end{abstract}

Palabras clave: Inteligencia Emocional / Adaptación Escolar / Validación empírica

\footnotetext{
* Mestre Navas J.M., es Doctor en Psicología. Profesor Asociado TC de la Universidad de Cádiz.Miembro del grupo de Investigación Inteligencia, Adaptación y Comportamiento. Su especialidad son las aplicaciones de la Psicología Básica en los ámbitos educativos y Humanidades. Líneas de investigación: evaluación, medida, e implicación de la I.E. en la adaptación humana y las necesidades de su desarrollo.

** Guil Bozal R., es Catedrática de la Escuela Universitaria de Psicología Social de la Universidad de Cádiz. Miembro del grupo de Investigación Inteligencia, Adaptación y Comportamiento.Siendo su especialidad las aplicaciones de la Psicología Social a los ámbitos educativos. Líneas de investigación:la I.E. en la adaptación socioescolar y sus necesidades de desarrollo

*** Mestre Moreno R., es Licenciada en Psicopedagogía, y está realizando su tesis doctoral sobre las aplicaciones de la I.E. como herramienta educativa para la formación de competencias emocionales.
} 


\begin{abstract}
The Educational System is one of the Emotional Intelligence (EI) implementation fields. From a few years back, the possible links between EI and students adaptation to the Educational System need some questions to be answered prior to the study of predictive validity in educational contexts. On one hand, some empirical data are needed to show we are faced with a novel construct and, if so, to prove whether EI is related to some adaptive criteria about educational adaptation. This research is only the beginning of a series of investigations by the authors aimed at showing that EI is a novel and autonomous construct, with independence from other constructs related to intelligence and personality. On the other hand, some outcomes related to adaptation are shown.
\end{abstract}

Key words: Emotional intelligence / School adaptation / empirical validation

\title{
Introducción
}

En los últimos años se ha ido trabajando y publicando más sobre la validez predictiva de la Inteligencia Emocional (IE) que sobre la naturaleza del modelo de habilidades cognitivas en el procesamiento de la información emocional de las 4 ramas (percepción, uso, facilitación y manejo de las emociones) de Mayer y Salovey (1997). Hasta ahora, algunos trabajos han verificado en cierta medida la validación de este modelo de habilidades (Lopes, Salovey y Straus, 2002, Mayer, Salovey, Caruso y Sitarenios, 2003 y Mestre, Guil, Extremera y Fernández-Berrocal, 2004). Para este propósito, se desarrolló el MSCEIT (Mayer, Salovey, Caruso Emotional Intelligence Test); un instrumento de evaluación de la IE basado en el modelo que comentamos. Sin embargo, algunos autores piensan que esta validación obedece más a cómo el MSCEIT está desarrollado y construido en diferentes tareas cognitivas de diversas índole (ocho secciones que cada dos de ellas miden una de las 4 ramas del modelo) que a una validación factorial (Thingujam, 2002 y Zeidner, Matthews y Roberts, 2001). El presente trabajo es un intento por acercarnos a la naturaleza de la IE a través de un estudio que verifique la naturaleza de la IE y su supuesta capacidad predictiva en el ámbito social y personal. Es decir, estamos ante un constructo de inteligencia o, por el contrario, ante uno de personalidad, o podemos estar ante un constructo realmente novedoso aunque relacionado con ambos términos de una manera no implícita (esto es que los índices de correlación no están por encima de 0,6). Desde nuestro punto de vista, creemos que esta tercera vía es posible a raíz de los datos presentados en estudios como los de Bracket y Mayer (2003) y Lopes, Salovey y Straus (2002) y los que adelantamos en este trabajo.

OBJETIVOS:

1. Determinar la posible naturaleza novedosa de la IE medida con el MSCEIT utilizando medidas de inteligencia y de personalidad.

2. Determinar la capacidad predictiva de la IE, incluyendo inteligencia y personalidad, con respecto a variables criterios como los índices de amistad en clase y la capacidad de reconocimiento de emociones. 


\section{Metodología}

\section{Participantes}

La muestra está formada por 127 sujetos $\left(50,4 \%\right.$ de mujeres) entre alumnos de $3^{\circ}$ $(52,8 \%)$ y de $4^{\circ}$ de secundaria $(47,2 \%)$ siguiendo una estrategia de muestreo aleatorio polietápico entre los alumnos de $3^{\circ}$ y $4^{\circ}$ de secundaria de la población de San Fernando (Cádiz). La decisión de utilizar este tipo de muestra es garantizarnos una mayor hetereogenidad de los sujetos de investigación, pues a estas edades (14-16 años) se encuentran, por ley, en los centros educativos.

\section{Procedimiento}

El diseño está basado en un estudio correlacional (no experimental por tanto) usando la variable de inteligencia emocional (MSCEIT) como variable sometida a estudio y de referencia y las medidas y de inteligencia general (IGF) y de personalidad (BFQ). El reconocimiento de emociones (DANVA), el índice de amistad en clase y la autoevaluación como variables criterios.

\section{Instrumentos}

\section{Variables predictoras}

El MSCEIT (Mayer, Salovey y Caruso, 2002): Esta prueba incluye ocho tareas para evaluar las cuatro ramas de IE: percibir, usar, entender y manejar las emociones. Además el MSCEIT tiene diferentes formas de puntuarse, de las que destacamos las valoraciones de los expertos (que será las que usemos en este trabajo) y las de consenso. Los valores de fiabilidad van desde 0,79 a 0,91 ; encontrándose un alto grado de convergencia entre las puntuaciones consensuadas y las de los expertos (Mayer, Salovey y Caruso, 2002; Mayer, Salovey, Caruso y Sitarenios, 2001). Para percibir emociones, se utilizan las secciones A (donde se presentan varias caras con una determinada expresión y se le pide al sujeto el grado adecuado de 1 a 5 de esas expresiones a cada una de las 5 emociones de una lista presentada al caso) y E (dinámica parecida a la anterior pero sustituyendo las caras por imágenes y que el sujeto identifica de una lista de emociones valorando de 1 a 5 cada una de ellas según identifique una imagen con una determinada emoción). Para usar emociones se integran los valores de las secciones B (donde al sujeto se le pregunta el grado de utilidad de un estado de humor para diferentes situaciones, Vg. “¿Qué estado de ánimo sería útil sentir cuando preparamos unos adornos nuevos y emotivos para una fiesta de cumpleaños?") y F (donde se les pide que evalúen de 1 a 4 el grado de semejanza de diferentes estados de humor con situaciones como Vg. "Imagina que te sientes culpable porque olvidaste visitar a un buen amigo que tiene una enfermedad grave. Al mediodía, te das cuenta de que olvidaste por completo ir a verlo al hospital"). Para comprender emociones se integran los valores de las secciones $\mathrm{C}$ (donde el sujeto elige una opción entre 5 posibilidades de preguntas como $\mathrm{Vg}$. "Una mujer amaba a una persona y luego se sintió segura ¿Qué ocurrió en medio?, a) se dio cuenta de que la otra persona también la amaba, b) decidió no expresar sus sentimientos, 
c) su amor se desvaneció, d) le dijo a la otra persona que la amaba y e) el amor que sentía le dio seguridad)" y la sección G (de una dinámica parecida a la anterior, Vg. "Una sorpresa triste lleva a ; a) desilusión, b) asombro, c) enojo, d) miedo y e) pena"). Finalmente, para manejar o regular emociones se integran las valoraciones de las secciones D (donde el sujeto valora de 1 a 4 el grado de eficacia de diferentes acciones para situaciones planteadas como: "María se levantó sintiéndose bastante bien. Había dormido estupendamente, se encontraba muy descansada y no tenía ningún tipo de inquietud o preocupación ¿Cómo ayudaría cada una de estas acciones a mantener su estado de ánimo?") y H (Parecido al anterior, se les pregunta al sujeto la eficacia de diferentes acciones sobre una determinada situación, Vg.: "A Lisa le va todo bien. Mientras los demás han recibido críticas por su trabajo, Lisa ha conseguido un ascenso y un aumento bastante bueno. Sus hijos están muy contentos y van muy bien en el colegio, su matrimonio es estable y muy feliz. Lisa empieza a sentirse muy orgullosa de sí misma y con la tentación de presumir sobre su vida a sus amigos ¿En qué medida sería eficaz cada una de las siguientes respuestas para mantener sus relaciones?"). El MSCEIT es corregido por la editorial MHS (Toronto, Canadá) de dos formas: a) por la puntuación otorgada por los expertos y b) la otorgada por consenso (utilizando la moda de las respuestas dadas por la muestra). Para este estudio fueron utilizadas las otorgadas por los expertos.

La inteligencia fue medida por el IGF (Yuste, 1998). Es una prueba estandarizada con diferentes niveles. Así el alumnado de $4^{\circ}$ de secundaria realizó el nivel M (medio) y los universitarios el nivel S (superior). Además, ambos niveles proporcionan los siguientes valores tales como el valor total de inteligencia general; el valor de la inteligencia no verbal (de la suma de las escalas manipulativas del test que son: razonamiento abstracto (considerado por muchos como un factor que está en la base de toda conducta inteligente) y la aptitud espacial (aptitud espacial, capacidad del sujeto para resolver problemas de tipo espacial y de figuras incompletas); el valor de la inteligencia verbal, compuesto por las subescalas de aptitud numérica (capacidad para resolver problemas de origen numérico) y razonamiento verbal (aptitud para entender y comprender problemas de tipo lingüístico). Además de estos valores, también son tenidos en cuenta la rapidez (tiempo que ha tardado en terminar la prueba), el número de respuestas contestadas y la eficacia (porcentaje de aciertos sobre las respuestas contestadas). La fiabilidad encontrada para el valor total en esta muestra fue de un alpha $=0,8132$.

El BFQ (Big Five Questionnary; Caprara, Barbaranelly y Borgogni, 1995): Es una prueba de personalidad basada en el modelo de los cinco grandes. Está baremada para muestras españolas y tiene la ventaja sobre el NEO-PIR (McCrae y Costa, 1997) de estar más adaptada para la población estudiantil española y tener menos ítems (Mestre, 2003). Esta prueba, aporta variables de estudio como dinamismo (alpha $=0,68)$, dominancia $($ alpha $=0,66)$, cooperación (alpha $=0,60)$, cordialidad $(\mathrm{alpha}=0,62)$, escrupulosidad (alpha $=0,71)$, perseverancia (alpha $=0,76)$, control de las emociones $($ alpha $=0,79)$, control de los impulsos (alpha 0,78), apertura a la cultura (alpha $=0,67)$, apertura a la experiencia $($ alpha $=0,64)$ y distorsión (subescala de sinceridad) (alpha $=0,79)$. Cada dos de estas subescalas forma cada uno de los 5 grandes. Así Energía es la suma de dinamismo y dominancia. Afabilidad es la de cooperación y cordialidad. Tesón de perseverancia y escrupulosidad. Estabilidad Emocional es la suma de control de emociones y de control de impulsos. Finalmente, Apertura Mental es la suma apertura a la cultura y de apertura a la experiencia. Normalmente, se usan los 5 grandes. Sin embargo cuando se usan las 10 subescalas del BFQ en vez de las 5 
generales el nivel de información de cómo la personalidad afecta a la IE es mucho mayor. Según Mestre (2003), dentro de una misma dimensión de cada una de los 5 grandes hay diferencias respecto a la medida de la IE. Así las personas perseverantes son más emocionalmente inteligentes que los escrupolosos. En el mismo sentido ocurre en otras dimensiones: cooperación que cordialidad; dinamismo que dominancia; control de emociones que control de impulsos; $y$, apertura a la cultura sobre apertura a la experiencia. Además según los autores del test, una distorsión positiva puede ser índice de una actitud triunfalista y una distorsión muy negativa síntomas de bajo autoconcepto. Para este estudio, optamos por utilizar sólo las 10 variables de personalidad, sin incluir las 5 dimensiones. Para verificar si la tendencia señalada por Mestre (2003) se mantiene utilizando una medida de tarea cognitiva de la IE (MSCEIT) en vez de una escala de percepción de IE.

\section{Variables criterios}

Los siguientes instrumentos se usaron como variables criterios para conocer qué aspectos de la personalidad, de la inteligencia o de la inteligencia emocional tienen una mayor influencia en la aceptación social, en la autovaloración personal y en la capacidad de reconocer las emociones.

ÍNDICES de AMISTAD: Donde el alumnado respondió a un cuestionario donde debía elegir a tres compañeros/as de su clase y responder a 21 cuestiones relacionadas con el funcionamiento personal y social del el sujeto elegido. Para este trabajo hemos seleccionado el número de veces que ha sido elegido un alumno y la puntuación media total otorgadas por los compañeros (valorándose entre 1 - poco- a 9 -mucho-). Ejemplos de estos ítemes son: ¿es sensible con los sentimientos y asuntos de los demás?, ¿Maneja bien el estrés?, o ¿es difícil relacionarse con él? (este ítem se recodifica posteriormente para los análisis). Se anularon 4 de los ítemes dada su baja fiabilidad. La fiabilidad final para los 17 ítems restantes fue de alpha $=0,8039$.

AUTOEVALUACIÓN: Es una escala con los mismos ítems que las utilizadas en las valoraciones de los índices sociométricos, pero en este caso en vez de para los compañeros elegidos para valorase a sí mismos sobre su funcionamiento personal y social dentro del aula. Al igual que el anterior se mide de 1 a 9 a lo largo de 21 ítems. Se eliminaron los mismos 4 ítemes que en el anterior y la fiabilidad final alpha fue de 0,7807.

DANVA (Diagnostic Analysis of Nonverbal Accuracy, Nowicki, 2000). Es un test de tareas cognitivas que consiste en reconocer o percibir adecuadamente 24 expresiones faciales distribuidas en 4 categorías (felicidad, tristeza, miedo y enojo) y una puntuación total para el reconocimiento de las emociones y para cada una de ellas. Además para cada imagen se incluye una grabación de audio con un determinado tono de voz. Para este trabajo la fiabilidad alpha del DANVA fue de 0,76 .

\section{Análisis y discusión de los resultados}

Los primeros resultados que anotamos son las correlaciones encontradas entre las diferentes variables de la IE con las de Inteligencia y Personalidad tal y como mostramos en la tabla 1 (para las relaciones con las medidas de inteligencia) y tabla 2 (para las de personalidad). 
TABLA 1: Correlaciones entre las puntuaciones otorgados por los expertos en el MSCEIT y las variables aportadas por el IGF $(* * * p<0,001 ; * * p<0,01$ y $* p<0,05)$.

\begin{tabular}{|l|c|c|c|c|c|}
\hline $\begin{array}{c}\text { Medidas } \\
\text { del } \\
\text { IGF }\end{array}$ & $\begin{array}{c}\text { MSCEIT } \\
\text { Percibir } \\
\text { Punt. } \\
\text { Expertos }\end{array}$ & $\begin{array}{c}\text { MSCEIT } \\
\text { Usar } \\
\text { Punt. } \\
\text { Expertos }\end{array}$ & $\begin{array}{c}\text { MSCEIT } \\
\text { Comprender } \\
\text { Punt. } \\
\text { Expertos }\end{array}$ & $\begin{array}{c}\text { MSCEIT } \\
\text { Manejar } \\
\text { Punt. } \\
\text { Expertos }\end{array}$ & $\begin{array}{c}\text { MSCEIT } \\
\text { Total } \\
\text { Punt. } \\
\text { Expertos }\end{array}$ \\
\hline IGF TOTAL &, 204 &, $369^{* * *}$ &, $339^{* *}$ &, $288^{* *}$ &, $446^{* * *}$ \\
\hline I No Verbal &, 103 &, $275^{* *}$ &, $238^{* *}$ &, $250^{*}$ &, $321^{* *}$ \\
\hline I Verbal &, $262^{*}$ &, $376^{* * *}$ &, $412^{* * *}$ &, $307^{* *}$ &, $489^{* * *}$ \\
\hline Raz. Abstr. &, 101 &, $289^{* * *}$ &, $286^{* *}$ &, $228^{*}$ &, $322^{* *}$ \\
\hline Apt. Espac. &, 148 &, 168 &, 102 &, 172 &, $242^{*}$ \\
\hline Raz. Verbal &, $203 *$ &, $457^{* * *}$ &, $499^{* * *}$ &, $364^{* * *}$ &, $549^{* * *}$ \\
\hline Apt. Numér. &, 202 &, 150 &, 180 &, 124 &, $259^{*}$ \\
\hline Efectiv. (\%) &, 173 &, $360^{* * *}$ &, $335^{* *}$ &, $281^{* *}$ &, $422^{* * *}$ \\
\hline
\end{tabular}

Tal y como muestran estos resultados, la IE como la definen Mayer y Salovey (1997) parece encontrar ciertos estándares de Inteligencia, en concreto está relacionada con la Inteligencia Verbal; tal y como ya apuntaron en anteriores ocasiones (Mayer, 2001; Mayer y Salovey, 1993 y 1995; Mayer, Caruso y Salovey, 2002 y Salovey, Mayer, Caruso y Lopes, 2001). La medida de la primera rama (percibir emociones) no mostró relaciones de importancia con las medidas de inteligencia, salvo con el razonamiento verbal, y que implica a lo sumo saber cómo se etiquetan las emociones, es decir, es más una cuestión de conocimiento que de habilidad. Sin embargo, en el resto de las ramas (usar, comprender y manejar las emociones) parecen encontrase más vinculaciones con aptitudes mentales más relacionadas con la inteligencia cristalizada (Gc) que con la inteligencia fluida (Gf) de los sujetos, pues son las subescalas de la Inteligencia Verbal del IGF las que se correlacionan significativamente más que con las no verbales (Gf). En este sentido, los estándares a los que se refieren estos autores no siguen, según estos indicios, las líneas (factores) similares a los modelos factoriales de inteligencia (Mestre, 2003) sino más bien, y sólo en algunos aspectos, siguen comportamientos similares a los de inteligencia cristalizada (Fox y Spector, 2000; Hedlund y Sternberg, 2000).

Aún así, podemos empezar a confirmar que la IE medida con el MSCEIT y descrita desde el modelo de habilidades cognitivas, tiene ciertas vinculaciones empíricas con habilidades mentales relacionadas con las aptitudes verbales, que con aspectos o rasgos biológicamente predeterminados (Zeidner, Roberts y Matthews, 2001) aunque no lleguen al criterio de 0,6 como indican Mayer, Salovey, Caruso y Sitarenios (2003). Por lo que aún puede ser cuestión discutible lo novedoso del constructo, si bien no queremos extendernos sobre ello sí podemos señalar que autores como Neisser (1976) ya indicaban que nuestra capacidad de procesamiento depende esencialmente del desarrollo de las habilidades específicas necesarias para ejecutar una determinada tarea (en este caso emocionales), y que dicha capacidad no está limitada ni por las características estructurales ni por las funcionales del sistema de 
procesamiento. Este tipo de resultados nos anima a desarrollar y perfeccionar un modelo explicativo de cómo las personas aprenden y usan dichas habilidades para ser eficaces ante tareas de tipo emocional (Mestre, Palmero y Guil, 2004).

Por su parte, tal y como se reflejan los resultados en la tabla 2, la personalidad parece tener también alguna vinculación significativa con las medidas de IE aportadas por el MSCEIT, pero están muy por debajo de los criterios de 0,6 o más que sugieren Mayer et al. (2003).

TABLA 2: Correlaciones entre las puntuaciones otorgados por los expertos en el MSCEIT y las variables aportadas por el BFQ de personalidad $\left(* * * p<0,001 ;{ }^{* *} p<0,01\right.$ y $\left.{ }^{*} p<0,05\right)$.

\begin{tabular}{|l|c|c|c|c|c|}
\hline \multicolumn{1}{|c|}{$\begin{array}{c}\text { Medidas } \\
\text { del } \\
\text { BFQ }\end{array}$} & $\begin{array}{c}\text { MSCEIT } \\
\text { Percibir } \\
\text { Punt. } \\
\text { Expertos }\end{array}$ & $\begin{array}{c}\text { MSCEIT } \\
\text { Usar } \\
\text { Punt. } \\
\text { Expertos }\end{array}$ & $\begin{array}{c}\text { MSCEIT } \\
\text { Comprender } \\
\text { Punt. } \\
\text { Expertos }\end{array}$ & $\begin{array}{c}\text { MSCEIT } \\
\text { Manejar } \\
\text { Punt. } \\
\text { Expertos }\end{array}$ & $\begin{array}{c}\text { MSCEIT } \\
\text { Total } \\
\text { Punt. } \\
\text { Expertos }\end{array}$ \\
\hline Dinamismo &, 061 &, $239^{*}$ &, 026 &, $228^{*}$ &, 153 \\
\hline Dominancia &, 032 &, 031 &, 055 &, 038 &, 027 \\
\hline Cooperación &, 146 &, $297^{* *}$ &, $254^{*}$ &, $486^{* * *}$ &, $393^{* * *}$ \\
\hline Cordialidad &, 101 &, 183 &, 037 &, $208^{*}$ &, 138 \\
\hline Escrupulosidad &,- 124 &, 049 &,- 105 &,- 014 &,- 087 \\
\hline Perseverancia &, 228 &, $253^{*}$ &, $283^{* *}$ &, $305^{* *}$ &, $321^{* *}$ \\
\hline Control Emoc. &, 020 &,$- 248^{*}$ &, 051 &,- 035 &,- 041 \\
\hline Control Impuls. &,- 096 &,- 013 &,- 069 &, 002 &,- 075 \\
\hline Apert. Cultura &, 073 &, $299^{* *}$ &, 170 &, $272^{* *}$ &, $282^{* *}$ \\
\hline Apert. Exper. &, 073 &, 088 &, 032 &, 182 &, 155 \\
\hline Distorsión &,$- 427^{* * *}$ &,$- 243^{*}$ &,$- 266^{*}$ &, 120 &,$- 394^{* * *}$ \\
\hline
\end{tabular}

De este modo, la considerable relación encontrada entre el rasgo cooperación con estas medidas de IE (especialmente con la rama de manejo de emociones, $r=0,486 ; p<0,001$ ), puede ser interpretado como que las personas cooperativas tienden a desarrollar habilidades relacionadas con el uso adaptativo de las emociones motivadas por el interés por ser eficaces en sus relaciones con el grupo. Por lo que, en cierto sentido la IE puede ser considerada como un tipo de Inteligencia Social (IS) como fue definida a priori por Salovey y Mayer (1990) ya que este tipo de personas desarrollan aptitudes cognitivas que inciden positivamente en la empatía, el manejo de conflictos, habilidades de negociación y liderazgo (Izard, 2001 y Lazarus y Lazarus, 1994).

Asimismo, aspectos de la personalidad como el dinamismo, la perseverancia y la apertura a la cultura parecen incidir positivamente en el desarrollo de habilidades relacionadas con la IE. Con esto, no se debe concluir que la IE descrita por Mayer y Salovey (1997) es una estructura cuya naturaleza está relacionada con la personalidad sino que más bien estamos ante algunos aspectos de la personalidad que están relacionadas con el desarrollo de 
ciertas habilidades cognitivas en el manejo adaptativo de las emociones. Así, como señalan Brody y Ehrlichman (1998) son numerosos los aspectos de la inteligencia vinculadas teórica y empíricamente con la personalidad, sin que por ello sea necesario incluir un constructo dentro del otro.

Finalmente, también es interesante el comportamiento de la subescala de distorsión. Salvo la rama de manejo de emociones las demás obtuvieron relaciones negativas significativas. La puntuación total del MSCEIT $(r=-0,394 ; P<0,001)$ con la distorsión puede estar relacionado con el hecho de que las personas con una distorsión negativa (una valoración de la personalidad por debajo de lo esperado) son los que menos puntuación obtienen en el test de IE. Como señalamos en el apartado de instrumentos, una distorsión demasiada baja puede ser un síntoma de baja autoestima (Borgogni, Caprara y Barbaranelli, 1994). Este sentido, la relación negativa y significativa con la IE es esperada.

Finalmente, en la tabla 3 mostramos los índices de correlación entre las medidas de IE, inteligencia y personalidad con las tres variables criterios: reconocimiento de emociones, el número de veces elegido como preferido en clase y la autoevaluación.

Una de las consecuencias previsibles, al menos teóricamente, del manejo adaptativo de las emociones y de una alta competencia emocional es la aceptación y liderazgo social, más promovido por aquellos que se apoyan en el modelo de IE de Goleman (Abraham, 1999; Fowler, 1999 y Murphy, 2002) y en el de Bar-On (Greener, 1999 y Dawda y Hart, 2000) que por aquéllos basados en el modelo de Mayer y Salovey (1997) más prudentes a la espera de estudios de "alta calidad científica" (Salovey, Woolery y Mayer, 2001). Por nuestra parte, los resultados obtenidos en este estudio no reflejan con respecto al número de veces que un alumno/a es elegido como preferido por sus compañeros que las medidas de IE, personalidad e inteligencia puestas en juego tengan alguna relación significativa, salvo por la perseverancia $(r=0,275, p<0,01)$. En cuanto a los índices sociométricos, la puntuación total de la IE $(r=0,266, p<0,05)$ y el uso de las emociones del MSCEIT $(r=0,249, p<0,05)$ mostraron relaciones significativas con la puntuación media total de los índices sociométricos obtenidos por los alumnos/as elegidos. Sin embargo, no predice más que algunos rasgos de personalidad como el dinamismo $(r=0,268, p<0,01)$ y el control de las emociones $(r=-0,210$, $p<0,05)$. Es decir, las personas emprendedoras, sociables y extrovertidas son más aceptadas por sus compañeros y aquéllas que se destacan por su poca capacidad para controlar las emociones (especialmente, la ira) son menos consideradas por el resto. Como vimos en la tabla 2, el dinamismo guardaba cierta relación con algunas de las subescalas del MSCEIT (usar y manejar emociones), por lo que es posible que personas con este rasgo hayan desarrollado las habilidades necesarias para manejar las emociones propias y la de los demás de forma adaptativa. El valor de distorsión (escala de sinceridad del BFQ, una puntuación baja es interpretable como bajo autoconcepto -Borgogni, Caprara y Barbaranelli, 1994-) refleja, como en los anteriores casos, una relación a considerar con las diferentes medidas, en el sentido que las personas que peor se consideran a sí mismos tienden a "venderse" peor entre los compañeros; aunque esta reflexión debe ser considerada como intuitiva.

En cuanto, a las medidas de autovaloración, sólo las medidas de personalidad se relacionaron significativamente con éstas, en el caso del dinamismo $(r=0,353, p<0,001)$, control de las emociones $(r=0,267, p<0,01)$ y perseverancia $(r=0,221, p<0,05)$. Estos resultados apoyan la sugerencia de Mestre (2003) de que hay rasgos de personalidad más emocionalmente competentes que otros dentro de una misma dimensión de los 5 grandes. 
TABLA 3: Correlaciones entre las variables de IE, Inteligencia y personalidad y las variables criterios: veces elegido, índice sociométricos, autovaloración y reconocimiento de emociones $(* * * p<0,001 ; * * p<0,01 \mathrm{y} * p<0,05)$.

\begin{tabular}{|l|c|c|c|c|}
\hline \multicolumn{1}{|c|}{ Medidas tomadas } & $\begin{array}{c}\text { Número de } \\
\text { veces elegido }\end{array}$ & $\begin{array}{c}\text { Puntuación } \\
\text { Media total de } \\
\text { sociométricos }\end{array}$ & $\begin{array}{c}\text { Valoración total } \\
\text { Autoevaluación }\end{array}$ & $\begin{array}{c}\text { DANVA total } \\
\text { Reconoc. de } \\
\text { emociones }\end{array}$ \\
\hline MSCEIT percibir &,- 037 &, 156 &, 158 &, 136 \\
\hline MSCEIT usar &, 133 &, $249^{*}$ &,- 030 &, $303^{* *}$ \\
\hline MSCEIT comprender &, 140 &, 179 &, 095 &, 199 \\
\hline MSCEIT manejar &, 090 &, 147 &, 132 &, $205^{*}$ \\
\hline MSCEIT total &, 127 &, $266^{*}$ &, 115 &, $288^{* *}$ \\
\hline IGF TOTAL &, 136 &, 076 &, 133 &, $369^{* * *}$ \\
\hline Intelig. No Verbal &, 075 &, 089 &, 109 &, $341^{* *}$ \\
\hline Intelig. Verbal &, 170 &, 057 &, 133 &, $309^{* *}$ \\
\hline Raz. Abstracto &, 088 &, 072 &, 049 &, $310^{* *}$ \\
\hline Apt. Espacial &,- 053 &,- 062 &, 098 &, $235^{* *}$ \\
\hline Raz. Verbal &, 137 &, 088 &, 141 &, $377^{* * *}$ \\
\hline Apt. Numérica &, 170 &,- 020 &, 081 &, 111 \\
\hline Efectividad &, 153 &, 076 &, 131 &, $357^{* * *}$ \\
\hline Dinamismo &, 098 &, $268^{* *}$ &, $353^{* * *}$ &, 021 \\
\hline Dominancia &, 177 &,- 174 &, 186 &, 059 \\
\hline Cooperación &, 140 &, 171 &, 223 &, 022 \\
\hline Cordialidad &, 091 &, 046 &, 192 &,- 011 \\
\hline Escrupulosidad &, 160 &, 001 &, 105 &, 042 \\
\hline Perseverancia &, $275^{* *}$ &, 112 &, $221^{*}$ &, 140 \\
\hline Control de emoc. &, 024 &,$- 210^{*}$ &, $267^{* *}$ &,- 058 \\
\hline Control de impuls. &,- 117 &, 012 &,- 103 \\
\hline Apertura cult. &,- 004 &,- 041 &, 186 \\
\hline Apertura experien. &, 156 &, 128 &, 088 \\
\hline Distorsión &,$- 244^{*}$ &, 138 &,- 064 \\
\hline
\end{tabular}

De nuevo, encontramos que las medidas de IE no parecen predecir un adecuado ajuste personal cuando se utilizan medidas percibidas y no criterios objetivos. En estudios recientes (Mestre, Guil, Lopes, Salovey y Gil-Olarte, en prensa) con criterios objetivos la IE parece consolidar su capacidad predictiva. Concretamente en los análisis de 
regresión los alumnos con más partes por conflictividad en el centro son los que menos puntuación obtuvieron en el MSCEIT. Estos resultados se han mostrado consistentes incluso controlando la influencia de la personalidad (medida con el BFQ) y la inteligencia (IGF).

Volviendo a este estudio y sin embargo, las medidas puestas en relación con el DANVA (reconocimiento de emociones) registró que las medidas de inteligencia y las de inteligencia emocional (salvo percibir y comprender) se relacionaron significativamente y positivamente con este test. El hecho de ausencia de significación entre la primera rama del MSCEIT (percepción) y el DANVA indica cierta incongruencia. Para ello, ya hay estudios que sugieren una prueba alternativa a la rama de percepción del MSCEIT dado los escasos resultados que en la investigación sobre validez predictiva sobre la IE, se está obteniendo con esta rama (Lopes, Brackett, Nezlek, Schütz, Sellin y Salovey, 2004). Sin embargo, las de personalidad no obtuviron relaciones significativas con el DANVA. Desde nuestro punto de vista, saber identificar una emoción es una habilidad relacionada con las aptitudes mentales en general (como podemos observar en las relaciones significativas obtenidas entre el DANVA y las medidas proporcionadas por el test de inteligencia IGF), pero la cuestión es que parece que no basta con eso para desarrollar comportamientos emocionalmente competentes.

\section{Conclusiones}

Si nos atenemos al primer objetivo, la naturaleza de la IE parece estar más cercana de las aptitudes mentales que de los rasgos de personalidad. En parte, se debe a la propia naturaleza de la medida de la IE utilizada. Las correlaciones entre IGF y MSCEIT son lo suficientemente altas para considerar que las personas con más capacidad e inteligencia verbal juegan con cierta ventaja cuando se les evalúan con el MSCEIT. Con este matiz, habría que aclarar cómo la IE se vincula a las aptitudes mentales que se cristalizan durante el ciclo vital de una persona. Y, si principalmente, es una habilidad desarrollada a partir de las aptitudes mentales cristalizadas (inteligencia cristalizada) que se van centrando en el manejo de las emociones; se necesitará explicar cómo ciertas personas con predominio de rasgos como el dinamismo, la perseverancia y la cooperación desarrollan dichas habilidades emocionales. Conviene destacar que la novedad del concepto es requerida para este tipo de estudios, pues algunos autores señalan que "estamos ante un vino viejo con una nueva botella" (Matthews, Zeidner y Roberts, 2002).

En cuanto al segundo objetivo, la IE no parece predecir, al menos a las edades que hemos manejado (13-17 años), una mejor aceptación social o valoración personal que lo que puedan hacer determinados rasgos de personalidad como el dinamismo o la perseverancia; por lo que, en un principio, estos datos parecen apoyar más las medidas basadas en los modelos mixtos, si bien éstas están basadas más en un perfil de competencias que en una verdadera medida de IE (Mestre, 2003).

Creemos que la IE es un constructo interesante, pero corre ciertos riesgos derivados de aspectos pocos relacionados con la Ciencia como la moda o el marketing. Pensamos que su futuro y estabilización pasa por desarrollar el modelo de habilidades desde el procesamien- 
to de la información y el conexionismo (Mestre, Palmero, Guil, 2004 y Mestre, Guil, Extremera, Berrocal y Lopes, 2002).

Una última reflexión nos queda por apuntar acerca del MSCEIT, una hipótesis alternativa a los resultados encontrados podrían deberse a las propiedades psicométricas de este instrumento; de tal manera que capta las habilidades mentales para ser emocionalmente inteligentes, pero a su vez, no garantiza que dichas aptitudes sean llevadas a la práctica.

\section{Reconocimientos}

Este trabajo ha sido realizado gracias a la subvención del MCyT español al proyecto BSO2000-1210 del grupo de investigación del plan andaluz de investigación HUM-554: Inteligencia, adaptación y comportamiento.

\section{Referencias bibliográficas}

Abraham, R. (1999). "EI in organizations: a conceptualization". Genetic, Social and General Psychology Monographs, 125 (2), 209-224.

Bracket, M. A. y Mayer, J. D. (2003). "Convergent, discriminant, and incremental validity of competing measures of Emotional Intelligence". Personality and Social Psychology Bulletin, 29, $1147-1158$.

Brody, N. y Ehrlichman, H. (1998). Personality psychology. The science of individuality. New York: Prentice Hall.

Caprara, G. V., Barbaranelli, C. y Borgogni, L. (1995). BFQ. Cuestionario "Big Five”. Manual. Madrid: TEA Ediciones.

Dawda, D. y Hart, S. D. (2000). "Assessing emotional intelligence: reliability and validity of the BarOn Emotional Quotient inventory (EQ-i) in university students". Personality and Individual Differences, 28 (4), 797-812.

Fowler, R. D. (1999). Managing a professional association. Psychologist Manager Journal, 3 (1), 57-69.

Fox, S. y Spector, P. E. (2000). "Relations of emotional intelligence, practical intelligence, general intelligence, and trait affectivity with interview outcomes: It's not all just ' $\mathrm{G}$ '”. Journal of Organizational Behavior, 21, Special Issue 2000, 203-220.

Greener, S. H. (1999). "The relationship between emotional predispositions, emotional decoding and regulation skills and children's prosocial behavior". Child Development, 59 (8-B), 4509.

Hedlund, J. y Sternberg, R. J. (2000). "Too many intelligences? Integrating social, emotional, and practical intelligence". En R. Bar-On y J. D. A. Parker (Coords.), The handbook of emotional intelligence: Theory, development, assessment, and application at home, school, and in the workplace (pp. 136-167). San Francisco, CA: Jossey-Bass.

Izard, C. E. (2001). "Emotional intelligence or adaptive emotions". Emotion, 1 (3), 249-257.

Lazarus, R. S. y Lazarus, B. N. (1994). Passion and reason. Oxford: Oxford University Press.

Lopes, P. N., Salovey, P., \& Straus, R. (2002). Emotional intelligence, personality, and the perceived quality of social relationships. Manuscrito pendiente de publicación.

Lopes, P. N., Brackett, M., Nezlek, J. B., Schütz, A., Sellin, I., y Salovey, P. (2004). "Emotional intelligence and social interaction". Personality and Social Psychology Bulletin, 30, 8, 1018-1034. 
Mayer, J. D. (2001). Emotion, intelligence, and emotional intelligence. En J. P. Forgas (Ed.). Handbook of affect and social cognition (pp. 410-431). Mahwah, NJ, USA: Lawrence Erlbaum Associates.

Mayer, J. D. y Salovey, P. (1993). "The intelligence of emotional intelligence". Intelligence, 17 (4), 433-442.

Mayer, J. D. y Salovey, P. (1995). "Emotional intelligence and the construction and regulation of feelings". Applied and Preventive Psychology, 4 (3), 197-208.

Mayer, J. D. y Salovey, P. (1997). “What is emotional intelligence?”. En P. Salovey y D. J. Sluyter (eds.). Emotional development and emotional intelligence: educational implications (pp. 3-34). New York: Basic Books.

Mayer, J. D., Salovey, P. y Caruso, D. (2002). MSCEIT. User's Manual. Toronto: MHS.

Mayer, J. D., Salovey, P., Caruso, D. R., y Sitarenios, G. (2001). "Emotional Intelligence as a Standard Intelligence". Emotion, 1, 3, 232-242.

Mayer, J. D., Salovey, P., Caruso, D. R., y Sitarenios, G. (2003). "Measuring Emotional Intelligence with the MSCEIT V2.0". Emotion, 3, 1, 97-105.

McCrae, R. R. y Costa, P. T. Jr. (1997). NEO-Pi-R: manual de aplicación. Madrid: TEA Ediciones.

Mestre, J. M. (2003). Validación empírica de una escala para medir la inteligencia emocional, desde un modelo mixto, en una muestra de estudiantes de la Bahía de Cádiz. Tesis Doctoral. Universidad de Cádiz: Servicios de publicaciones.

Mestre, J. M., Palmero, F. y Guil, R. (2004). Inteligencia Emocional: "Una Explicación Integradora desde los Procesos Psicológicos Básicos". En J. M. Mestre y F. Palmero (Coords.), Procesos Psicológicos Básicos: una guía académica para los estudios en Psicología y Psicopedagogía. Madrid: McGraw-Hill.

Mestre, J. M., Guil, R., Extremera, N. y Fernández Berrocal, P. (2004). "Validación del MSCEIT (Mayer, Salovey, Caruso Emotional Intelligence Test) en muestras españolas mediante el uso del programa LISREL 8.30". En libro de resúmenes del VII European Conference on Psychological Assessment. Málaga (España) 1-4 abril de 2004.

Mestre, J. M., Guil, R., Extremera, N., Fernández-Berrocal, P. y Lopes, P. N. (2002). "Emotional intelligence: an approximation from limited capacity on attention model of Kahneman". Dissertation Abstracts International, Section B/123, 3451. XI ISRE Conference, Cuenca.

Murphy, S. E. (2002). "Leader self-regulation: The role of self-efficacy and multiple intelligences". En S. E. Murphy (ed.). Leader self-regulation: the role of self-efficacy and multiple intelligences (pp. 163-186). Mahwah, NJ, US: Lawrence Erlbaum Associates.

Nowicki, S., Jr. (2000). Manual for the receptive tests of the Diagnostic Analysis of Nonverbal Accuracy 2. Atlanta, GA: Department of Psychology, Emory University.

Neisser, U. (1976). Cognition and reality: Principles and implications of cognitive psychology. San Francisco: Freeman.

Salovey, P. y Mayer, J. D. (1990). "Emotional intelligence". Imagination, Cognition and Personality, 9 (3), 185-211.

Salovey, P., Woolery, A. y Mayer, J. D. (2001). "Emotional Intelligence: conceptualization and measurement". En G. Fletcher y M. S. Clark (Eds.). Social Psychology (pp. 279-307). Londres: Blackwell.

Salovey, P., Mayer, J. D., Caruso, D. y Lopes, P. N. (2001). "Measuring emotional intelligence as a set of abilities with the MSCEIT". En S. J. López, y R. Snyder (Eds.), Handbook of positive psychology assessment (pp. 243-267). Washington, DC: American Psychological Association. 
Thingujam, N. S. (2002). "Emotional intelligence: What is the evidence?". Psychological Studies, $47(1-3)$.

Yuste, C. (1996). IGF. Inteligencia General Factorial. Madrid: Tea Ediciones.

Zeidner, M., Roberts, R. D. y Matthews, G. (2001). "Slow down, you move too fast: emotional intelligence remains an 'elusive' intelligence". Emotion, 1 (3), 265-275.

Fecha de recepción: 19-05-04

Fecha de revisión: 07-07-05

Fecha de aceptación: 19-10-05 